\title{
Plate stability under periodic load action, material of which obeys hereditary law of viscoelasticity
}

\author{
Elena Kosheleva ${ }^{1, a}$ \\ ${ }^{1}$ Moscow State University of Civil Engineering (National Research University) \\ Yaroslavskoe Shosse 26, Moscow, Russia
}

aelleok@mail.ru

Keywords: dynamic stability, rectangular plate, viscoelastic material.

Abstract. The problem of a rectangular plate dynamic stability is considered, the material of which obeys the hereditary deformation law. The differential equation solution for oscillations of a plate loaded with constant and variable load in the plate plane is considered as a series with separated variables. For the time function, integro-differential equations are obtained. The equation of critical frequencies is obtained. The effect of nuclear parameters on the position of the main region of dynamic instability is investigated.

\section{Problem Formulation}

Consider a rectangular plate loaded in its plane by alternating compressive forces uniformly distributed along the edges:

$$
N_{x}=N_{x 0}+N_{x t} \cos \theta t, \quad N_{x}=N_{x 0}+N_{x t} \cos \theta t
$$

In [1] a plate was considered, the material of which obeyed the linear creep law, which does not take into account the features of the work at the beginning of the process. Therefore, we use the hereditary Boltzmann-Volterra law with a weakly singular creep kernel to account for the viscoelastic properties of the material [2]:

$$
R(t-s)=A e^{-\beta(t-s)}(t-s)^{\alpha-1}(0<\alpha<1) .
$$

The presence of three parameters makes this kernel suitable for describing the behavior of many polymer materials that obey the hereditary deformation law. There is a technique for determining the parameters of the kernel [3]. Assuming $v$ as constant, we have the relationship between strains and distortion:

$$
\begin{aligned}
& \sigma_{x}=\frac{E}{1-v^{2}}\left(\varepsilon_{x}+v \varepsilon_{y}\right)-\frac{E}{1-v^{2}} \int_{0}^{t} R(t-\theta)\left(\varepsilon_{x}+v \varepsilon_{y}\right) d \theta, \\
& \sigma_{y}=\frac{E}{1-v^{2}}\left(\varepsilon_{y}+v \varepsilon_{x}\right)-\frac{E}{1-v^{2}} \int_{0}^{t} R(t-\theta)\left(\varepsilon_{y}+v \varepsilon_{x}\right) d \theta, \\
& \tau_{x y}=\frac{E}{2(1+v)} \gamma_{x y}-\frac{E}{2(1+v)} \int_{0}^{t} R(t-\tau) \gamma_{x y} d \theta
\end{aligned}
$$

The equation for the transverse vibrations of the plate has the form of:

$$
D\left(\nabla^{4} w-\int_{0}^{t} R(t-s) \nabla^{4} w d s\right)+\left(N_{x 0}+N_{x t} \cos \theta t\right) \frac{\partial^{2} w}{\partial x^{2}}+\left(N_{y 0}+N_{y t} \cos \theta t\right) \frac{\partial^{2} w}{\partial y^{2}}+m \frac{\partial^{2} w}{\partial t^{2}}=0,
$$

at $D=\frac{E h^{3}}{12\left(1-v^{2}\right)}$ - cylindrical stiffness, $E$ - instantaneous modulus of elasticity.

\section{Solution method}

We seek the solution of the equation in the form of 


$$
w=\sum f_{i}(t) X_{i}(x) Y_{i}(y) \text {. }
$$

The Bubnov-Galerkin method yields an equation for the determination of $f_{i}(t)$, which we transform to a dimensionless form:

$$
f^{\prime \prime}-\varepsilon \varphi \int_{0}^{\tau} R(\tau-s) f(s) d s+\varphi(1-2 \mu \cos \tau) f=0,
$$

where the dimensionless time is $\tau=\theta t, \omega$ is natural oscillation frequency of an unloaded plate,

$$
\varphi=\frac{\Omega^{2}}{\theta^{2}}, \varepsilon=\frac{\omega^{2}}{\Omega^{2}}, \Omega^{2}=\omega^{2}\left(1-\frac{N_{x 0} N_{2 *}+N_{y 0} N_{1 *}}{N_{1 *} N_{2 *}}\right), \mu=\frac{1}{2} \frac{N_{x t} N_{2 *}+N_{y t} N_{1 *}}{N_{1 *} N_{2 *}-N_{x 0} N_{2 *}-N_{y 0} N_{1 *}},
$$

$\Omega$ is natural oscillation frequency of a loaded plate, $N_{x 0}$ and $N_{y 0}$, - excitation factor, $N_{1 *}$ and $N_{2 *}$ are critical effort values $N_{x 0}$ and $N_{y 0}$ with their independent static action,

$$
\begin{aligned}
& N_{2 *}=\frac{I_{1}}{I_{4}} D, I_{1}=\int_{0}^{a} \int_{0}^{b}\left(\frac{d^{4} X}{d x^{4}} Y+2 \frac{d^{2} X}{d x^{2}} \frac{d Y^{2}}{d y^{2}}+\frac{d Y^{4}}{d y^{4}} X\right) X Y d x d y, I_{2}=\int_{0}^{a} \int_{0}^{b} X^{2} Y^{2} d x d y, \\
& I_{3}=-\int_{0}^{a} \int_{0}^{b} X \frac{d^{2} X}{d x^{2}} Y^{2} d x d y, I_{4}=-\int_{0}^{a} \int_{0}^{b} X^{2} \frac{d^{2} Y}{d y^{2}} Y d x d y, \omega^{2}=\frac{D I_{1}}{m I_{2}} .
\end{aligned}
$$

The coefficients in Eq. 6 affect its solutions. For a certain ratio of the coefficients these solutions can increase without bound and occupy the regions in the plane $\mu, \theta / 2 \Omega$. One of the main problems of the theory of dynamic stability [5] is the determination of regions of dynamic instability. The domains of unboundedly increasing solutions are separated from the instability regions by periodic solutions with periods $T$ and $2 T$. The most dangerous is the first region of instability, which can be found by looking for the solution of Eq. 6 in the form of a series with period $T$ :

$$
f(t)=\sum_{k=1,3,5}^{\infty}\left(a_{k} \sin \frac{k \tau}{2}+b_{k} \cos \frac{k \tau}{2}\right)
$$

Substituting Eq. 7 into Eq. 6 and transforming the integrals, we obtain the equation:

$$
\begin{aligned}
& \sum_{k=1}^{\infty}\left(-a_{k} \frac{k^{2}}{4} \sin \frac{k \tau}{2}-b_{k} \frac{k^{2}}{4} \cos \frac{k \tau}{2}\right)-\varepsilon \varphi \sum_{k=1}^{\infty}\left[\sin \frac{k \tau}{2}\left(a_{k} B_{k}+b_{k} A_{k}\right)+\cos \frac{k \tau}{2}\left(b_{k} B_{k}-a_{k} A_{k}\right)\right]+ \\
& \varphi \sum_{k=1}^{\infty}\left(a_{k} \sin \frac{k \tau}{2}+b_{k} \cos \frac{k \tau}{2}\right)-\mu \varphi \sum_{k=1}^{\infty} a_{k}\left(\sin \frac{k-2}{2} \tau+\sin \frac{k+2}{2} \tau\right) \\
& -\mu \varphi \sum_{k=1}^{\infty} b_{k}\left(\cos \frac{k-2}{2} \tau+\cos \frac{k+2}{2} \tau\right)=0,
\end{aligned}
$$

where

$$
A_{k}=\int_{0}^{\tau} R(x) \sin \frac{k x}{2} d x, B_{k}=\int_{0}^{\tau} R(x) \cos \frac{k x}{2} d x, x=\tau-s
$$

Equating the coefficients with the same $\sin \frac{k \tau}{2}$ and $\cos \frac{k \tau}{2}$, we obtain a system of linear equations with respect to $a_{k}$ and $b_{k}$ : 


$$
\begin{aligned}
& -\frac{1}{4} a_{1}-\varepsilon \varphi a_{1} B_{1}-\varepsilon \varphi b_{1} A_{1}+\varphi a_{1}+\mu \varphi a_{1}-\mu \varphi a_{3}=0, \\
& -\frac{1}{4} b_{1}-\varepsilon \varphi b_{1} B_{1}+\varepsilon \varphi a_{1} A_{1}+\varphi b_{1}-\mu \varphi b_{1}-\mu \varphi b_{3}=0, \\
& -\frac{k^{2}}{4} a_{k}-\varepsilon \varphi a_{k} B_{k}-\varepsilon \varphi b_{k} A_{k}+\varphi a_{k}-\varphi \mu\left(a_{k+2}+a_{k-2}\right)=0, \\
& -\frac{k^{2}}{4} b_{k}-\varepsilon \varphi b_{k} B_{k}+\varepsilon \varphi a_{k} A_{k}+\varphi b_{k}-\varphi \mu\left(b_{k+2}+b_{k-2}\right)=0, \\
& (k=1,3,5 \ldots)
\end{aligned}
$$

We compose of the coefficients with $a_{k}$ and $b_{k}$ the determinant, equating it to zero, we obtain the equation of critical frequencies:

$$
\left|\begin{array}{ccccc}
p^{2}+\varepsilon B_{1}-1-\mu & \varepsilon A_{1} & \mu & 0 & \ldots \\
-\varepsilon A_{1} & p^{2}+\varepsilon B_{1}-1+\mu & 0 & \mu & \ldots \\
\mu & 0 & 9 p^{2}+\varepsilon B_{3}-1 & \varepsilon A_{3} & \ldots \\
0 & -\mu & -\varepsilon A_{3} & 9 p^{2}+\varepsilon B_{3}-1 & \ldots \\
\ldots & \ldots & \ldots & \ldots & \ldots
\end{array}\right|=0 \text {, }
$$

The coefficients $A_{k}$ and $B_{k}$ are functions of time, therefore, the stability regions change with time. The functions $A_{k}$ and $B_{k}$, changing, approach the value of the integral with an infinite upper limit of integration. Then, starting from a certain time, the stability regions do not change their position, and the coefficients can be calculated by the following formulas:

$$
\begin{aligned}
& A_{k}=A \int_{0}^{\infty} x^{\alpha-1} e^{-\beta x} \sin \frac{k x}{2} d x=A \frac{\Gamma(\alpha)}{\left(\beta^{2}+\frac{k^{2}}{4}\right)^{\frac{\alpha}{2}}} \sin \left(\alpha \operatorname{arctg} \frac{k}{2 \beta}\right), \\
& B_{k}=A \int_{0}^{\infty} x^{\alpha-1} e^{-\beta x} \cos \frac{k x}{2} d x=A \frac{\Gamma(\alpha)}{\left(\beta^{2}+\frac{k^{2}}{4}\right)^{\frac{\alpha}{2}}} \cos \left(\alpha \operatorname{arctg} \frac{k}{2 \beta}\right),
\end{aligned}
$$

where $\Gamma(\alpha)$ is gamma function.

\section{Results}

Let us investigate the influence of the kernel parameters on the position of the instability regions. For this we solve Eq. 11. We consider the second approximation using the fourth-order determinant. The equation includes coefficients that affect the solution. These coefficients depend on the parameters of the kernel. For example, for the case of increasing $A$ with constant $\alpha$ and $\beta$, the coefficients $A_{k}$ and $B_{k}$ change in direct proportion to its value. Figure 1 shows the results of calculating the regions of instability in the plane $\mu, \frac{\theta}{2 \Omega}$ for values $\alpha=0.15, \beta=0.05, A=0.019-0.103$ [3]. It should be noted that with increasing $A$ the minimum value of the excitation coefficient 


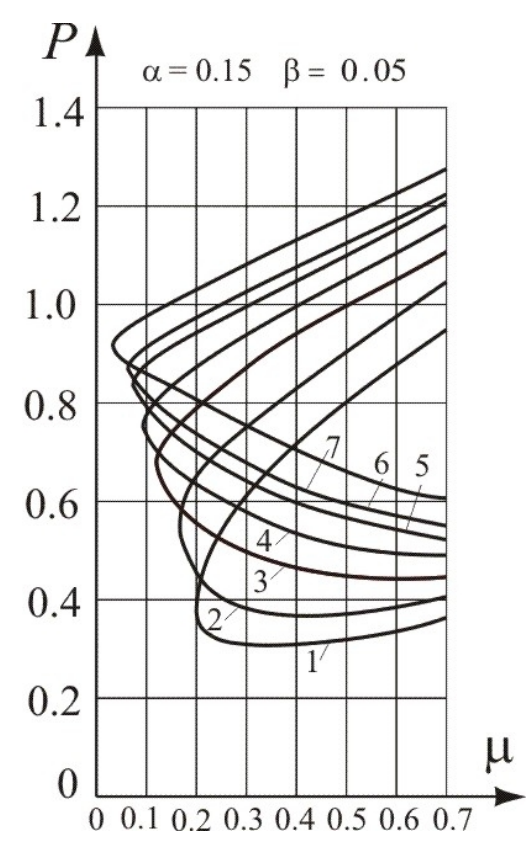

Fig.1. The boundaries of the main instability areas:

$$
\begin{aligned}
1-A=0.103 ; 2-A=0.083 ; \\
3-A=0.064 ; 4-A=0.051 ; \\
5-A=0.038 ; 6-A=0.032 ; \\
7-A=0.019 .
\end{aligned}
$$

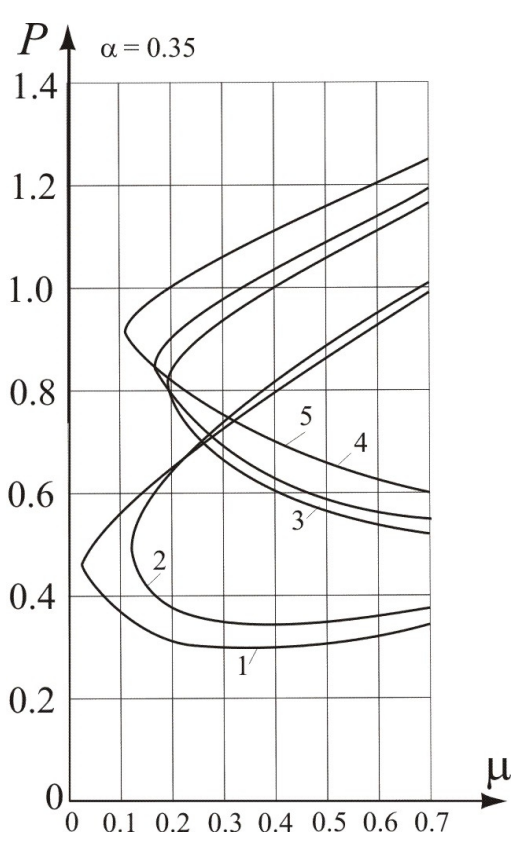

Fig.2. The boundaries of the main instability areas:

$1-A=0.5, \beta=7.69 ; 2-A=0.25, \beta=1.061$;

3- $A=0.1, \beta=0.0 ; 4-A=0.085, \beta=0.049$;

$$
\text { 5- } A=0.05, \beta=0.011 \text {. }
$$

$\mu$ increases, that is, the periodic components amplitudes of forces capable of causing a loss of dynamic stability increase, which corresponds to the move of the regions to the right along the $\mu$ axis. In this case, the frequency of the periodic load decreases, which corresponds to the displacement of the curves in side of smaller values at $p=\theta / 2 \Omega$. Let us consider the stability regions with $\alpha$ constant at variable values of $A$ and $\beta$. Figures 2 and 3 show the main regions of

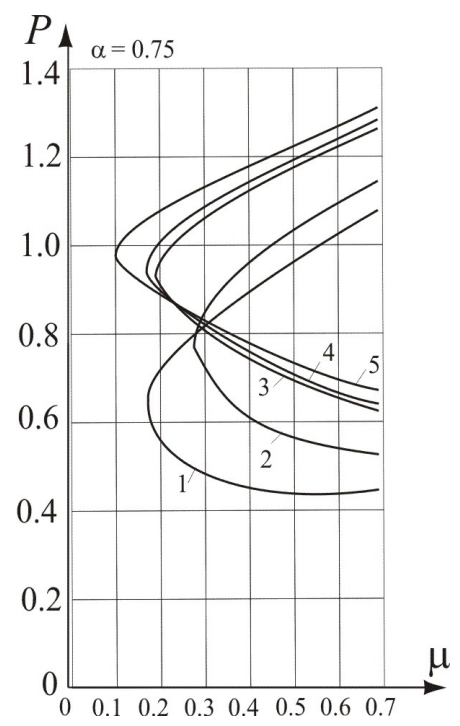

Fig.3. The boundaries of the main instability areas: $1-A=0.5, \beta=1.214$,

2- $A=0.5, \beta=0.482$,

3- $A=0.1, \beta=0.142$,

4- $A=0.085, \beta=0.1143$,

5- $A=0.05, \beta=0.056$, instability at $\alpha=0.35$ (Fig. 2) and $\alpha=0.75$ (Fig. 3) where the coefficients $A$ and $\beta$ increase. Note that $A$ varies from 0.05 to 0.5 . With the growth of $A$ and $\beta$ the graphs in the figures move downwards, toward the decrease of $p$, shifting simultaneously first to the right and then to the left, approaching the $p$ axis. Analogous displacements of the boundaries of dynamic instability regions were observed using a linear differential ratio of viscoelasticity [1] and the long modulus of elasticity was constant, and the relaxation rate varied from zero to an infinitely large value. The existing point of intersection of all curves [1] in this case at $\alpha=0.75$ is practically present, and disappears when $\alpha=0.35$. Thus, at a constant value of $\alpha$ the increase of $A$ and $\beta$ leads to a decrease in the frequency of the periodic load causing a loss of dynamic stability. The most dangerous for $\alpha=0.35$ is the region at $\beta=7.69$, since the curve closest approaches the $p$ axis. 


\section{Conclusion}

The effect of the parameters of the core of a viscoelastic material of a rectangular plate on the position of the main region of dynamic instability is investigated. It was found that with increasing $A$ the minimum value of the excitation coefficient $\mu$ increases, that is, the amplitudes of the periodic components of forces capable of causing a loss of dynamic stability increase, and at a constant value of $\alpha$ the increase in $A$ and $\beta$, which leads to a decrease in the frequency of the periodic load causing a loss of dynamic stability. It should be noted that viscoelastic behavior of structural elements is most characteristic for polymers and composites. Recently, articles have been published in which new results are presented on the investigation of viscoelastic properties of materials and the development of methods for calculating the corresponding structures [6 - 10 etc.]

\section{References}

[1] E.L. Kosheleva in: MATEC Web of Conferences 117, 00086 (2017)

[2] A.R. Rzhanitsyn: Creep theory. (Stroyizdat M., 1968).

[3] M.A. Koltunov in: Mechanics of polymers 4, p.483-497(1966)

[4] I.E. Triyanovky in: Vestnik MGU, 6, p.67-72(1970)

[5] V.V. Bolotin, Dynamic Stability of Elastic Systems (State publishing house of theoretic technical literature, 1956).

[6] N.Yu.Tsybin, R.A.Turusov, V.I.Andreev, Procedia engineering 153 ( 2016 )

[7] V.I. Andreev, A.S. Chepurnenko, B.M. Yazyev, Advanced Materials Research, 009 (2014)

[8] V.I. Andreev, A.S. Chepurnenko, B.M. Yazyev, Advanced Materials Research 1004-1005 (2014)

[10] V.I. Andreev, A.S. Chepurnenko, B.M. Yazyev, A.N. Beskopylny, MATEC Web of Conferences 67 (2016) No 06059 